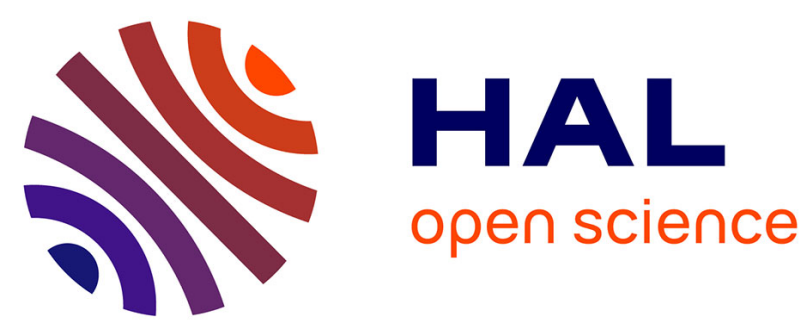

\title{
On the versatility of electronic structures in polymethine dyes
}

Simon Pascal, Alexandre Haefele, Cyrille Monnereau, Azzam Charaf-Eddin, Denis Jacquemin, Boris Le Guennic, Olivier Maury, Chantal Andraud

\section{- To cite this version:}

Simon Pascal, Alexandre Haefele, Cyrille Monnereau, Azzam Charaf-Eddin, Denis Jacquemin, et al.. On the versatility of electronic structures in polymethine dyes. SPIE Security + Defence, 2014, Amsterdam, Netherlands. 10.1117/12.2072624 . hal-01896789

\section{HAL Id: hal-01896789 \\ https://hal.science/hal-01896789}

Submitted on 24 Feb 2021

HAL is a multi-disciplinary open access archive for the deposit and dissemination of scientific research documents, whether they are published or not. The documents may come from teaching and research institutions in France or abroad, or from public or private research centers.
L'archive ouverte pluridisciplinaire HAL, est destinée au dépôt et à la diffusion de documents scientifiques de niveau recherche, publiés ou non, émanant des établissements d'enseignement et de recherche français ou étrangers, des laboratoires publics ou privés. 


\title{
On the Versatility of Electronic Structures in Polymethine Dyes
}

\author{
Simon Pascal, ${ }^{\mathrm{a}}$ Alexandre Haefele, ${ }^{\mathrm{a}}$ Cyrille Monnereau, ${ }^{\mathrm{a}}$ Azzam Charaf-Eddin, ${ }^{\mathrm{b}}$ Denis Jacquemin, ${ }^{\mathrm{b}}$ \\ Boris Le Guennic, ${ }^{\mathrm{c}}$ Olivier Maury ${ }^{\mathrm{a}^{*}}$ and Chantal Andraud ${ }^{\mathrm{a}^{*}}$ \\ ${ }^{a}$ Laboratoire de Chimie, CNRS UMR 5182, Université Lyon 1, Ecole Normale Supérieure de Lyon, \\ 46 allée d'Italie, 69364 Lyon Cedex 07 \\ ${ }^{\mathrm{b}}$ Laboratoire CEISAM, CNRS UMR 6230, Université de Nantes 2, 2 rue de la Houssinière, BP \\ 92208, 44322 Nantes Cedex 3, France. \\ ${ }^{\mathrm{c}}$ Laboratoire des Sciences Chimiques de Rennes, CNRS UMR 6226, Université de Rennes 1, 263 \\ avenue du Général Leclerc, 35042 Rennes Cedex, France. \\ *olivier.maury@ens-lyon.fr; chantal.andraud@ens-lyon.fr
}

\begin{abstract}
This article provides an overview of the photophysical behavior diversity of polymethine chromophores which are ubiquitous in biological imaging and material sciences. One major challenge in this class of chromophore is to correlate the chemical structure to the observed optical properties, especially when symmetry-breaking phenomena occur. With the constant concern for rationalization of their spectroscopy, we propose an extended classification of polymethine dyes based on their ground state electronic configuration using three limit forms namely: cyanine, dipole and bis-dipole. The chemical modifications of the dye and the influence of exogenous parameters can promote dramatic spectroscopic changes that can be correlated to significant electronic reorganization between the three-abovementioned forms. The deep understanding of such phenomena should allow to identify, predict and take advantage of the versatile electronic structure of polymethines.
\end{abstract}

Keywords: Polymethine, near-infrared dyes, cyanine, dipole, bis-dipole, spectroscopy, electronic absorption

\section{INTRODUCTION}

Polymethines are $\pi$-conjugated molecules featuring an odd number of $\mathrm{sp}^{2}$ carbon atoms. An incredible wide variety of polymethines have been reported since the 1850's,[1] with a length comprised between 1 and $13 \mathrm{C}$ atoms and with various electron-donating and/or withdrawing extremities resulting in the formation of neutral, positively or negatively charged dyes.[2-5] The most common derivatives possess a positive charge perfectly delocalized between electrondonating indole moieties through the conjugated bridge which confer the polymethine dyes their unique structural and optical properties. This ideal polymethine state is generally referred to as the cyanine state. It is accompanied by an equalization of the bond lengths and is characterized by sharp "solitonic" absorption and emission in the red, nearinfrared region, reminiscent of polyacetylene conductors. This last feature accounts for the craze for polymethines and their use in a vast range of applications such as biological imaging,[6-8] photovoltaic devices[9] or as electroluminescent switches.[10,11] In that framework, some of the most recent breakthroughs have been made in the field of nonlinear optics in the near infrared spectral range, where the usefulness of polymethines has clearly been demonstrated in the context of optical limiting,[12, 13] all-optical switching,[14-16] electro-optic modulation[17, 18] and biphotonic bioimaging applications.[19, 20] However, a major issue with polymethines is the difficulty to explain and predict the nature and magnitude of the changes in optical properties that take place upon structural variations such as: chain elongation,[21] functionalization[22] or under the influence of their environment.[23, 24] Therefore it appears necessary to get a better understanding of these structure-to-properties relationships using an appropriate model.

\section{CHEMICAL AND ELECTRONIC DEFINITIONS OF POLYMETHINE DYES}

\subsection{Chemical classification of polymethine dyes}

In the 60-70's, Dähne proposed a classification of organic unsaturated chromophores, distinguishing three classes, as depicted in Figure 1: aromatic cycles; polyenes with an even number of carbon atoms; polymethines with an odd number of carbon atoms.[25] Both aromatic and polymethine states possess a strong resonance and a bond length alternation 
(BLA) equal to zero.[26] Conversely, the polyene state is characterized by an alternation of single and double bonds along the $\pi$-conjugated backbone. This early classification attempts to correlate chemical structures and electronic properties but does not give account of the strong modifications of the electronic configuration for a given chemical structure under the influence of external parameters.[27] For instance, under certain circumstances, polymethine can display electronic/spectroscopic properties almost undistinguishable from those of polyenes, and vice-versa.
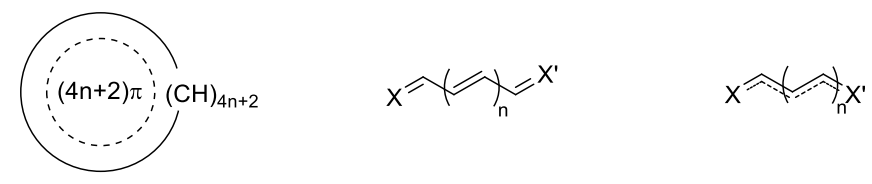

aromatic state

polyene state

polymethine state

Figure 1. Three classes of organic unsaturated compounds introduced by Dähne.

In their reference review, Behera et al. proposed a more accurate description of the diversity of the polymethine family. This classification however, was still based on exclusive structural considerations (Figure 2).[2] Thus, four classes were distinguished, according to their charge state: (i) cationic cyanines, (ii) anionic oxonols, (iii) neutral merocyanines and (iv) zwitterionic (charge separated) cyanines. This classification has been very popular among chemists because it is based on the schematic representation of the dye. But once again, it does not fully account for the variety of spectroscopic characteristics of polymethines: although these four families exhibit different structures, it is well established that they can all reach identical electronic properties.

$$
\left.R_{2} N+\right)_{n}{\stackrel{\oplus}{N H R_{2}}}^{x^{\Theta}}
$$

(i) cyanine<smiles>O=CC=CC(=O)O</smiles>

(ii) oxonol<smiles>[R16]N=CC=CC=O</smiles>

(iii) merocyanine<smiles>[R20]C=C1C(=O)C(C=[R6])=C1[O-]</smiles>

(iv) zwitterionic cyanine

Figure 2. Four families of polymethine dyes according to their charge introduced by Behera et al.[2]

\subsection{Electronic classification of polymethine dyes}

To tackle this drawback, a first alternative classification based on the electronic structure has emerged which provides a more accurate description and rationalization of their spectroscopic properties.[28-30] According to this model, highlighted in Figure 3, symmetrical and unsymmetrical polymethines can be stabilized under two different forms depending on their charge (de)localization. The dipole structure corresponds to a polarized form where one end of the polymethine possesses a higher electronic density than the other extremity. The dipole is characterized by a non-zero BLA and a particularly broad and solvatochromic linear absorption spectrum. In the case of an unsymmetrical chemical structure, this form can be stabilized under either neutral or zwitterionic configurations. Conversely, when the charge is perfectly delocalized through the polymethine bridge, the cyanine electronic structure is reached: the BLA vanishes and the linear absorption spectrum displays a typical intense, narrow and not solvatochromic transition. This classification allows explaining that a given structure can exhibit various electronic configurations hence spectroscopic properties under the influence of various external stimuli or chemical modifications, as illustrated by the upcoming selected examples. 


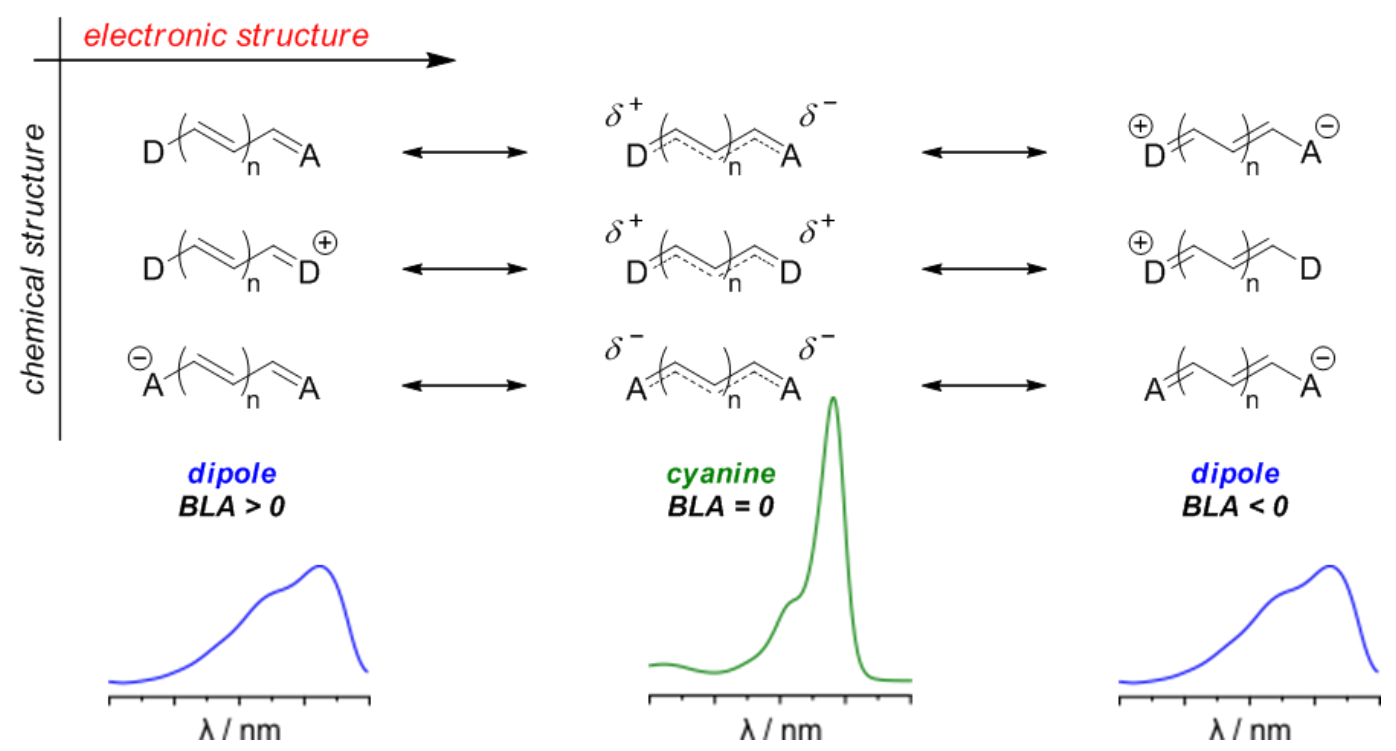

Figure 3. Resonance structures and absorption profiles of symmetrical and unsymmetrical polymethines.

\section{TRANSITIONS BETWEEN DIPOLE AND CYANINE ELECTRONIC CONFIGURATIONS}

The transition between cyanine and dipole forms has been punctually reported and appears to be sensitive and generally unexpected. This phenomenon occurs upon chemical modification or under the influence of exogenous parameters such as $\mathrm{pH}$ or polarity. The modulation of the electronic structure is always characterized by easily observable changes of the optical properties.

\subsection{Transition induced by chemical modification}

The pioneering Brooker experiment consists in increasing the length of a symmetrical cyanine dyes: a strong bathochromic shift of the cyanine transition is observed upon elongation, by about $100 \mathrm{~nm}$ per vinyl moieties, until a critical limit is reached where a broadening of the absorption band is observed.[31] This behavior was eventually explained by Tolbert in a benchmark studies,[21] as a consequence of loss of the cyanine character due to symmetry breaking for longer chains (Peierls-type distortions). This hypothesis was further confirmed by theoretical calculations, which demonstrated that the elongated polymethine dyes adopt a dipolar electronic configuration.[32-34]

In 1997, Würthner and co-workers studied the variation of the electro-donating strength of the donor extremity in the case of a series of unsymmetrical polymethines (Figure 4).[35] The dyes that incorporate weak donor groups were shown to display broad dipole-type absorption whereas increasing the strength of the donor resulted in a red shift of the absorption band featuring ultimately the sharp solitonic profile characteristic of a cyanine electronic structure. This transition from dipole-to-cyanine was explained on the basis of the increased delocalization along the $\pi$-conjugated skeleton resulting in the equalization of the electronic density at both extremities and a vanishing of the BLA. Consequently an unsymmetrical polymethine structure can present a symmetrical cyanine-type electronic configuration.<smiles>[R]C=C1C(=O)N(C(C)(C)C)C(=O)C(C#N)=C1C</smiles><smiles>[R][K]</smiles><smiles>CCCOc1ccc(C)cc1</smiles><smiles>COc1ccc(I)s1</smiles><smiles>CCCCNc1ccc(I)cc1</smiles><smiles>CCCCNc1ccc(I)s1</smiles>

Figure 4. Unsymmetrical polymethines incorporating various donors reported by Würthner et al.[35] 


\subsection{Transition induced by solvent polarity}

This dipole-to-cyanine transition has also been described in the case of a single unsymmetrical polymethine (Figure 5) under the influence of the solvent.[36] When increasing the solvent polarity, a strong bathochromic and hyperchromic shift is observed which can be rationalized as followed. In less polar solvent, the transition is broad and typical of a dipolar structure. In a more polar solvent the absorption becomes sharper and presents the characteristic profile of a cyanine electronic structure. Finally, as the solvent polarity still increases (case of $\mathrm{CH}_{3} \mathrm{CN}$ ), the absorption band becomes less intense and broader. This last behavior corresponds to a charge separation in highly polar solvent and illustrates the transition from a cyanine to a zwitterionic dipole (Figure 3). This unique example describes a double transition from a dipole-to-cyanine and then from a cyanine-to-(charge separated) dipole. Similarly, multiple studies about unsymmetrical polymethines close to the cyanine limit underline the possibility to stabilize these compounds under their cyanine structure by influence of solvent polarity.[18, 29, 37-40]

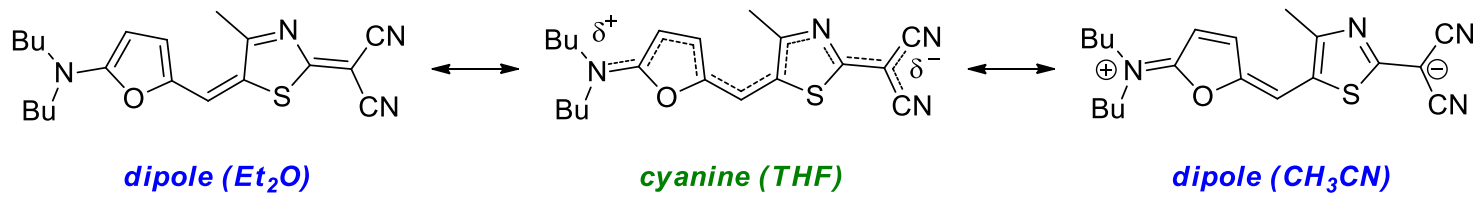

Figure 5. Various electronic configuration reached by an unsymmetrical polymethine under the influence of the solvent polarity.[36]

The influence of the solvent polarity has also been explored in the case of symmetrical polymethines. A representative example is described by Lepkowicz in 2004 using a dye containing 11 conjugated carbon atoms (Figure 6).[41] In low polarity solvent this polymethine exhibits a characteristic cyanine absorption band but as the polarity increases a broad band appears at lower energy. The authors rationalized this behavior by an increased contribution in more polar solvent of the asymmetric electronic configuration. Using the present classification, this behavior illustrates a cyanine-to-dipole transition induced by the solvent.

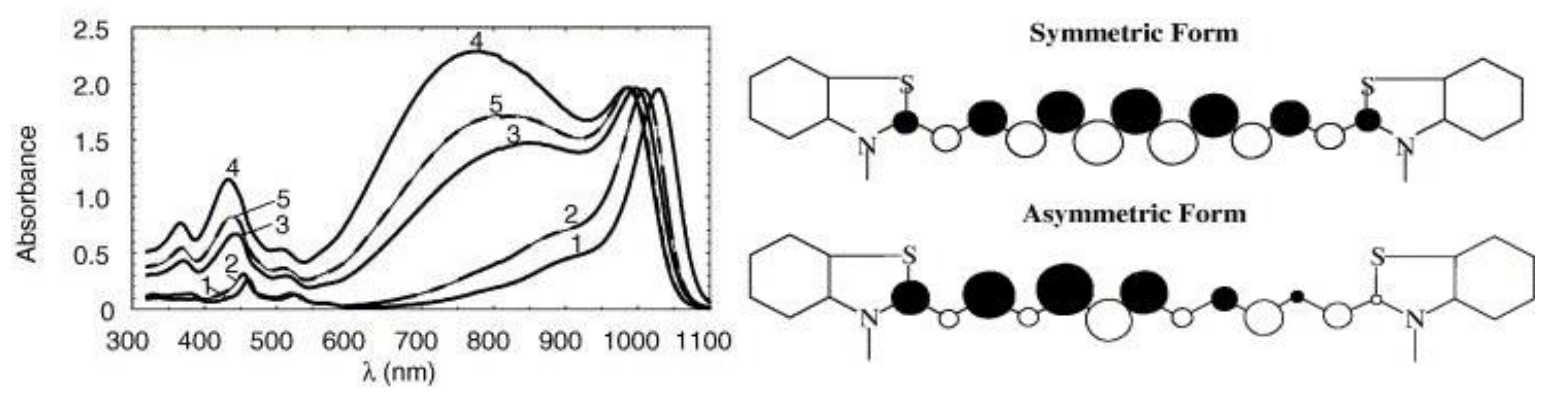

Figure 6. Absorption spectra of undecamethine reported by Lepkowicz et al. (left) in $o$-dichlorobenzene (1), dichloromethane (2), ethanol (3), acetonitrile (4) and methanol (5). Determined symmetric and asymmetric forms (right). Figure adapted from ref. [41].

\subsection{Transition induced by protonation}

The protonation is also an elegant way to promote dipole-to-cyanine transition. In 2000, Cooper and co-workers reported the $\mathrm{pH}$-dependent optical properties of an unsymmetrical pentamethine with one non-alkylated indole extremity (Figure 7).[42] In basic conditions, this dye displays a broad absorption spectrum (dipole type) whereas in acidic medium, the transition is dramatically red-shifted and becomes sharper and more intense (cyanine type). This cyanine electronic structure is simply reached because of the protonation of the free-base indole extremity. A positive charge is then generated and is perfectly delocalized along the conjugated skeleton. Several analogous polymethines have been reported more recently: these $\mathrm{pH}$ probes took advantage of a non-fluorescent dipolar form whereas the protonated cyanine form exhibited an intense red fluorescence.[43-46] 


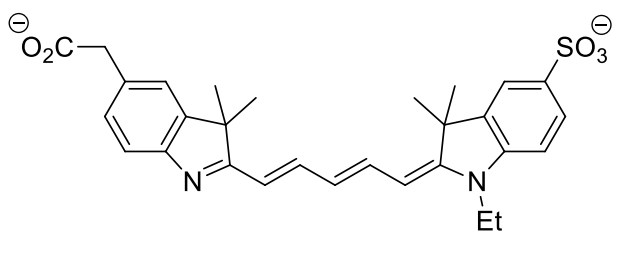

dipole

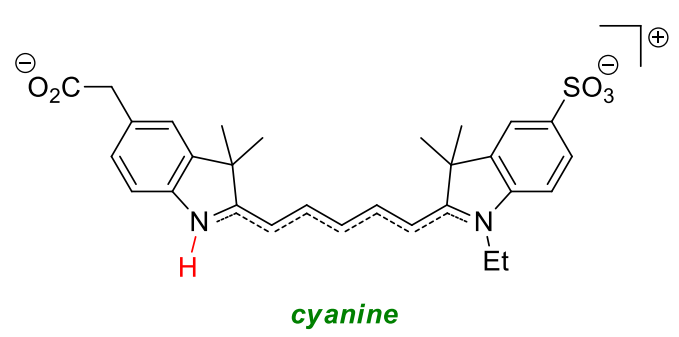

Figure 7. pH-sensitive pentamethine dye developed by Cooper et al.[42]

\subsection{Transition induced by ion-pairing}

Finally, our group demonstrated that the optical properties of symmetrical cationic heptamethine can be strongly influenced by the nature of the counter-anion.[24, 47] A strong ion-pair is formed with small counter-anions in a nondissociative solvent or at the solid state resulting in a localization of the cationic charge at one extremity of heptamethine $\mathbf{D D}[\mathbf{B r}]$ and consequently a polarization of the conjugated backbone. Consequently, this loss of symmetry favours a dipole electronic structure, with a non-zero BLA in the X-ray structure and a broad absorption profile in apolar solvent (Figure 8). Increasing the dissociative character of the solvent or the bulkiness of the counter-anion prevents the ionpairing phenomena which restores the cyanine electronic structure. This study showed that ion-pairing phenomena can also trigger the cyanine-to-dipole transition.

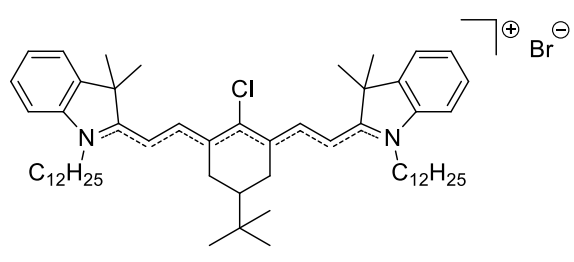

cyanine $\left(\mathrm{CH}_{2} \mathrm{Cl}_{2}\right)$

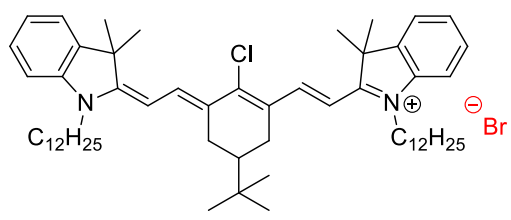

dipole (toluene)

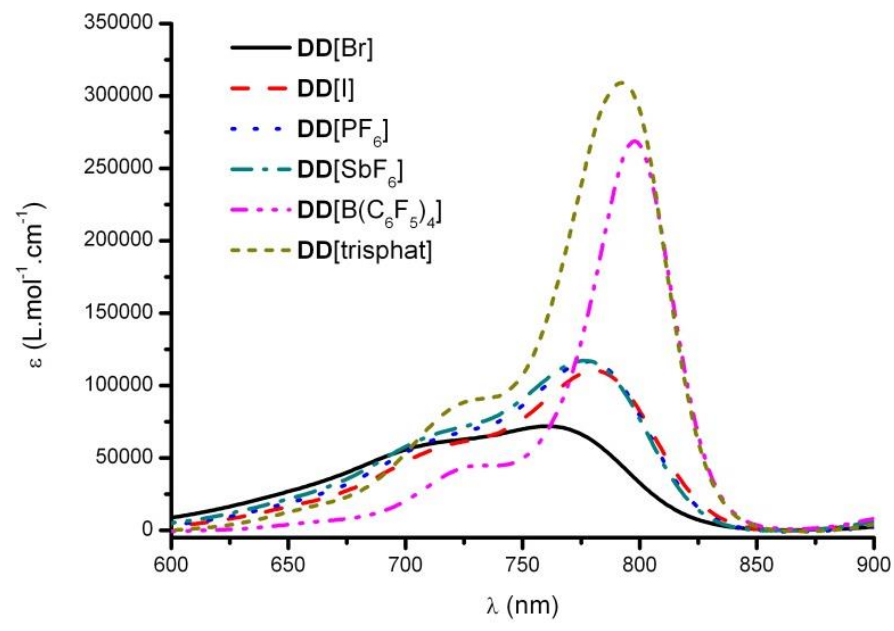

Figure 8. Influence of ion-paring effect on the absorption spectra of heptamethine dye DD[Br] in toluene.[47]

In conclusion, all these examples illustrate the electronic versatility of symmetrical or unsymmetrical polymethine dyes, under the influence of chemical modification or exogenous parameters and highlighted the interest of the electronic classification for the interpretation and rationalization of the observed optical modifications. However it appears that the dipole and cyanine models are not always sufficient to explain all the observed phenomena and that this first classification remains incomplete.

\section{THE BIS-DIPOLE ELECTRONIC CONFIGURATION: A PARTICULAR CASE OF CHARGE LOCALIZATION}

\subsection{Preliminary study: the amino-heptamethine}

In particular the modification of the central position of polymethine dyes has raised questions during the last decade. Peng and co-workers reported in 2005 the optical properties of a symmetrical heptamethine featuring a secondary amine at the central position.[22] The replacement of the usual central chlorine atom by an amine led to a dramatic blue shift of 
the electronic absorption as illustrated in Figure 9 (using analogous DD-Cl and DD-NHPr). In the case of DD-NHPr, both absorption and emission are broad with a larger Stokes shift. Immediately this class of molecules has become very popular for bio-imaging applications due to their high quantum yield and larger Stokes shift.[48-51] However, the influence of the central functionalization on optical properties remains not fully understood: although the cyanine character is clearly lost, no clear explanation was proposed, the authors putting forward the hypothesis of a charge transfer at the excited state. Therefore we have undertaken a systematic study of the role of the central substitution of heptamethines in order to explain this loss of cyanine character.[52]
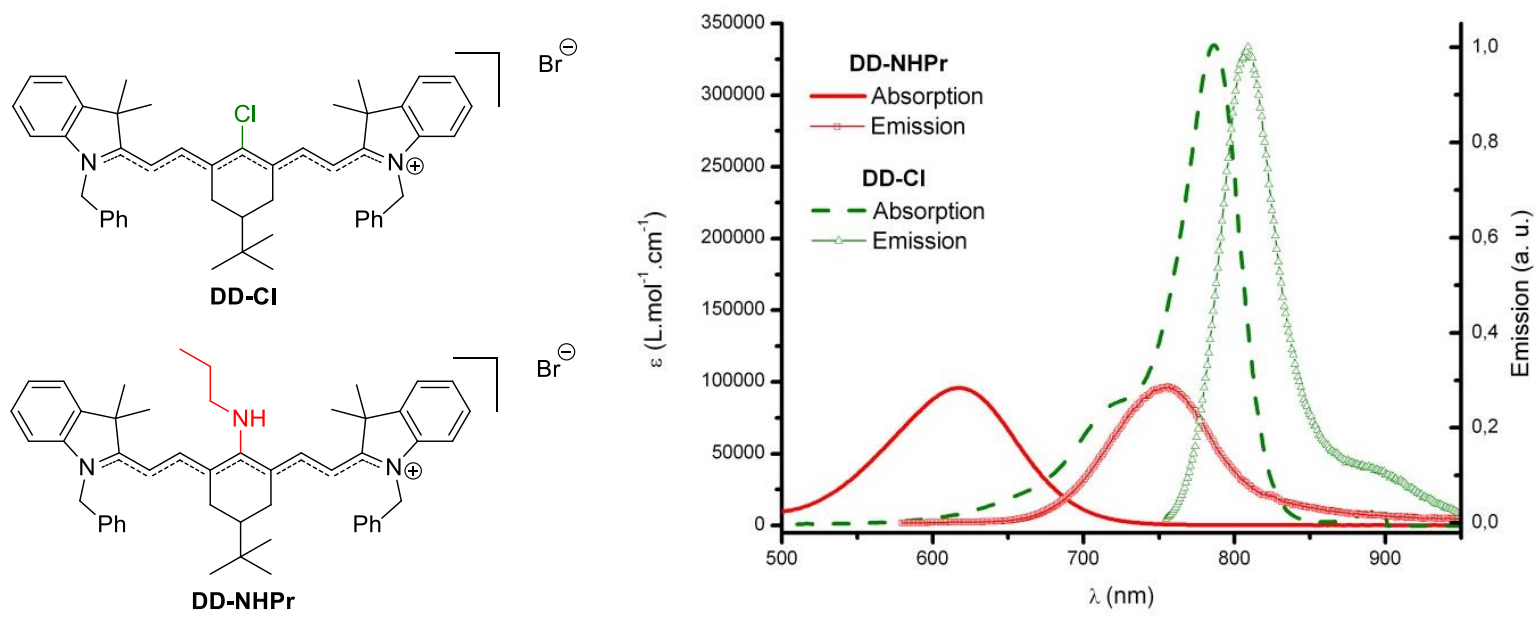

Figure 9. Structure and optical properties in methanol of heptamethines DD-Cl and DD-NHPr.

\subsection{Electronic absorption of polymethines substituted at the central position}

A series of 17 heptamethine dyes (Figure 10) incorporating various central functionalization was thus prepared by nucleophilic substitution.[30, 53-56] These compounds incorporate central functions such as halogens (DD-Cl, DD-Br), amines (DD-NEt 2 , DD-Pip, DD-NHPr), imines (DD=NPr, DD=NPh), ketone (DD=O), ester (DD-OAc), (thio)phenol (DD-OPh, DD-SPh)... Such substituants allow screening the influence of different electro-donating strength, particularly in the case of the aniline series (DD-NO $\left.\mathbf{N O}_{2}, \mathbf{D D}-\mathbf{A n C F}_{3}, \mathbf{D D}-\mathbf{A n H}, \mathbf{D D}-\mathbf{A n O M e}\right)$.

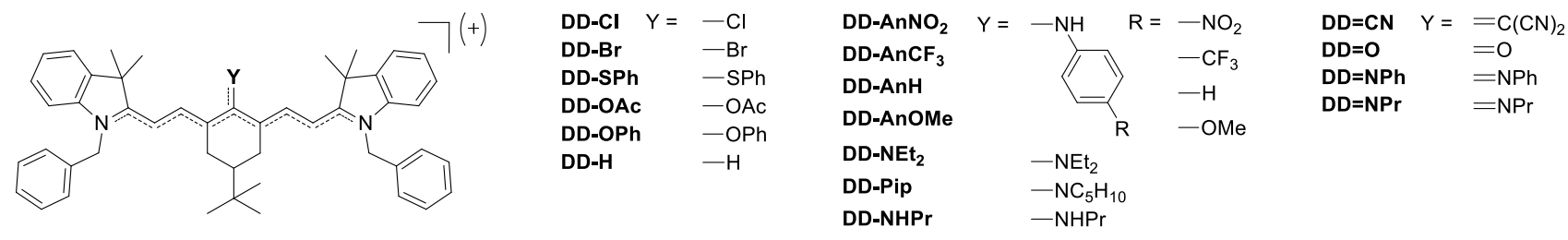

Figure 10. Series of heptamethines substituted on the central position.

The absorption spectra of the heptamethine were recorded in dichloromethane solution and are presented on Figure 11 (left). A first observation is that the central functionalization has a strong influence on the transition wavelengths and allows tuning the absorption from the UV-visible region towards the near-infrared region (350-850 nm). Two behaviors are highlighted: some heptamethines present characteristic cyanine transition localized around $800 \mathrm{~nm}$ (DD-SPh, DD-Br, DD-Cl, DD-OAc, DD-H, DD-OPh) whereas the other functionalized heptamethines exhibit broad and less intense absorption band, blue-shifted up to $350 \mathrm{~nm}$. Considering the localization of the band and the nature of the central substituent, it appears that the more electro-donating the latter is, the more blue-shifted the absorption is. 

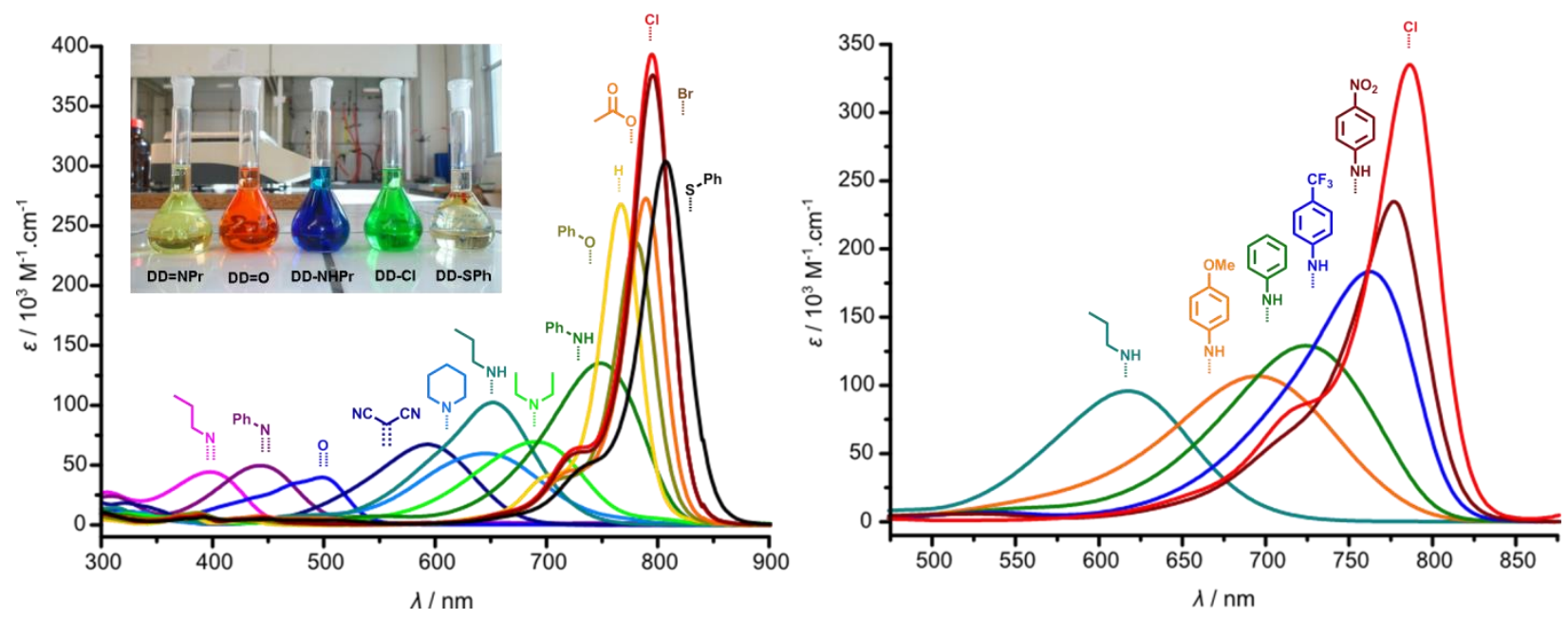

Figure 11. Electronic absorption spectra of heptamethines in dichloromethane (left) and in methanol (right). The inset is a picture of selected compounds in dichloromethane solutions (ca. $0.04 \mathrm{mg} \cdot \mathrm{L}^{-1}$ ).

In order to rationalize these observations, we proposed a model (Figure 12), where the electro-donating central substituent contributes in stabilizing the cationic charge of the heptamethine at the central position. The progressive localization of the charge is responsible for the shift of the electronic structure away from the cyanine configuration towards a new electronic configuration that we defined as bis-dipole. In the extreme case of heptamethines with a central double bond $(\mathbf{D D}=\mathbf{C N}, \mathbf{D D}=\mathbf{O}, \mathbf{D D}=\mathbf{N P h}, \mathbf{D D}=\mathbf{N P r})$, the substituent can be depicted in a limit form, in which an anionic central substituent (i.e. with the highest electro-donating effect) faces the centered cationic charge, leading to the most blue-shifted absorption bands.

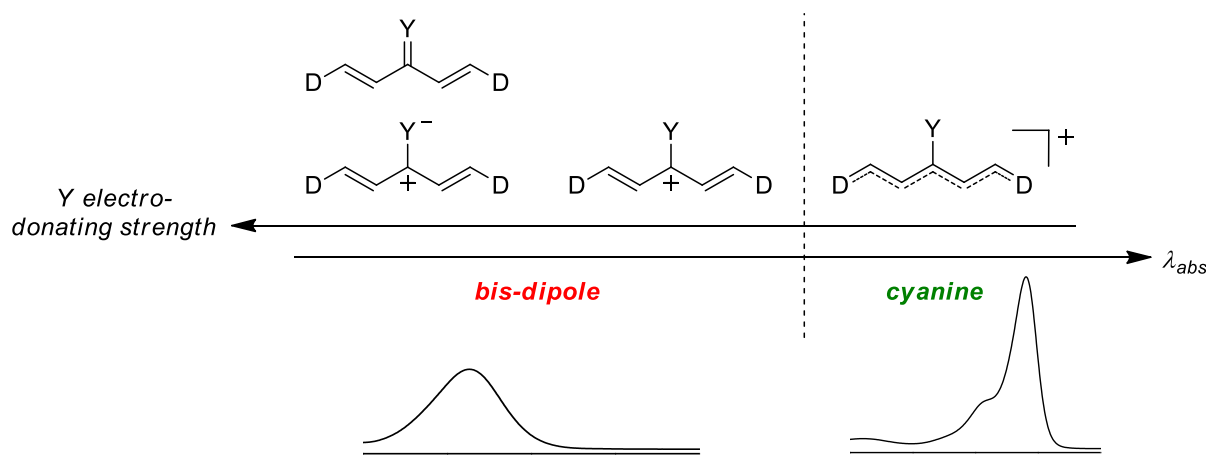

Figure 12. Model describing the electronic structure and absorption shape of a polymethine as the cationic charge is progressively localized towards the central position.

To test this hypothesis, we synthesized a series of aniline substituted dyes DD-AnX $\left(\mathrm{X}=\mathrm{NO}_{2}, \mathrm{CF}_{3}, \mathrm{H}, \mathrm{OMe}\right)$ whose absorption is located at the frontier between the two electronic behaviours. Consequently, minor alterations of the electron-donating ability of the aniline substituent are sufficient to lead the chromophore to adopt either of these two configurations. The absorption spectra of the aniline series (Figure 11, right) show that the decrease of the electron density on the aniline moieties $\left(\mathrm{NO}_{2}>\mathrm{CF}_{3}>\mathrm{H}>\mathrm{OMe}\right)$ lead to a hyperchromic and bathochromic shift of the transition, bridging the gap between the bis-dipole and the cyanine forms. The existence of the bis-dipole electronic configuration and the bis-dipole-to-cyanine transition were also unambiguously confirmed by the linear correlations between the absorption energy and ${ }^{13} \mathrm{C}$ NMR, BLA or local charge (the last two parameters being obtained by DFT calculations).[52] This correlation between optical observation and parameters representative of the electronic delocalisation further validates the choice of the electronic rather than structural classification of polymethines. 


\section{CONCLUSION}

In conclusion, this article demonstrated that polymethine dyes can be best described according to a classification using not two but three electronic configurations (Figure 13): (i) the symmetrical cyanine with a perfectly delocalized charge, (ii) the unsymmetrical dipole with a charge localized at one extremity and (iii) the bis-dipole with a charge localized at the central position. This classification allows an easier rationalization of the spectroscopy of this whole chromophore family: for a given structure and environment (solvent, $\mathrm{pH}$, counter-ion...) the electronic configuration of the dye can shift from one to the other limit electronic configuration, or more realistically, consists in a combination of these three forms with variable ponderations. In a more general perspective, we believe that these three-forms model can not only be helpful to improve one's understanding of the versatile optical properties of polymethines but can also constitute a useful toolbox to develop new responsive-probes or switches.

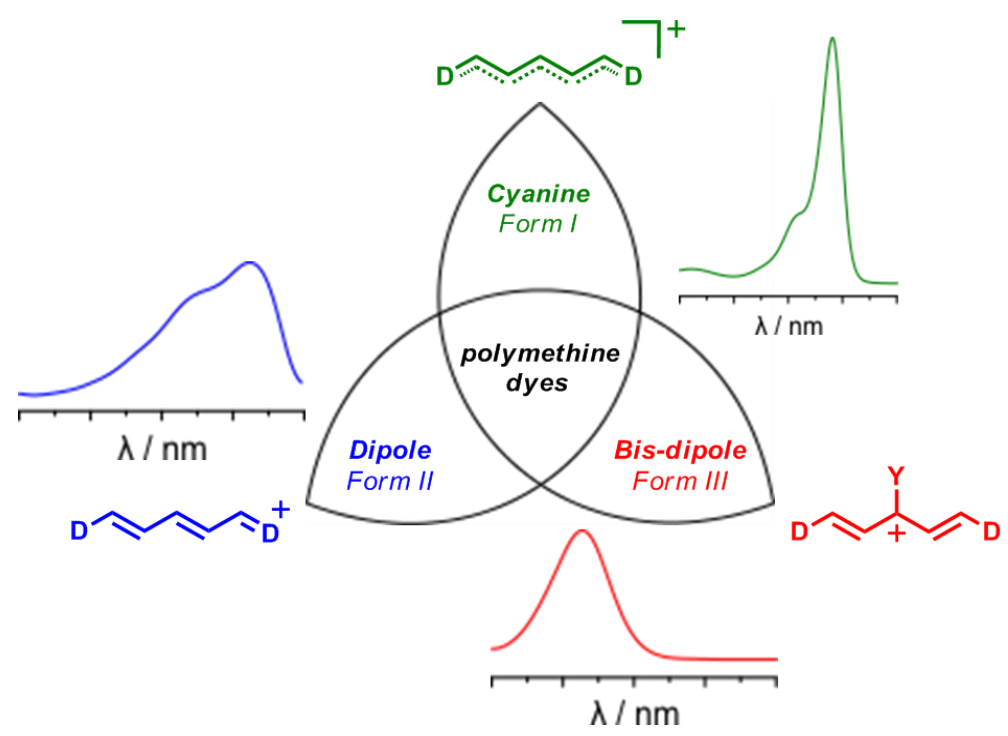

Figure 13. Complete electronic classification of polymethine dyes.

\section{REFERENCES}

[1] C. H. G. Williams, "Researches on Chinoline and its Homologues," Trans. Roy. Soc. Edinburgh, 21(03), $377-$ 401 (1856).

[2] A. Mishra, R. K. Behera, P. K. Behera et al., "Cyanines during the 1990s: A Review," Chemical Reviews, 100(6), 1973-2012 (2000).

[3] A. V. Kulinich, and A. I. Aleksandr, "Merocyanine dyes: synthesis, structure, properties and applications," Russian Chemical Reviews, 78(2), 141 (2009).

[4] P.-A. Bouit, E. Di Piazza, S. Rigaut et al., "Stable Near-Infrared Anionic Polymethine Dyes: Structure, Photophysical, and Redox Properties," Organic Letters, 10(19), 4159-4162 (2008).

[5] Q. Bellier, N. S. Makarov, P.-A. Bouit et al., "Excited state absorption: a key phenomenon for the improvement of biphotonic based optical limiting at telecommunication wavelengths," Physical Chemistry Chemical Physics, 14(44), 15299-15307 (2012). 

Journal, 3(3), 506-515 (2008).

[7] G. Qian, and Z. Y. Wang, "Near-Infrared Organic Compounds and Emerging Applications," Chemistry - An Asian Journal, 5(5), 1006-1029 (2010).

[8] Z. Guo, S. Park, J. Yoon et al., "Recent progress in the development of near-infrared fluorescent probes for bioimaging applications," Chemical Society Reviews, 43(1), 16-29 (2014).

[9] P.-A. Bouit, D. Rauh, S. Neugebauer et al., "A "Cyanine-Cyanine" Salt Exhibiting Photovoltaic Properties," Organic Letters, 11(21), 4806-4809 (2009).

[10] A. Pertegás, D. Tordera, J. J. Serrano-Pérez et al., "Light-Emitting Electrochemical Cells Using Cyanine Dyes as the Active Components," Journal of the American Chemical Society, 135(48), 18008-18011 (2013).

[11] S. Seo, S. Pascal, C. Park et al., "NIR electrochemical fluorescence switching from polymethine dyes," Chemical Science, 5(4), 1538-1544 (2014).

[12] P.-A. Bouit, G. Wetzel, G. Berginc et al., "Near IR Nonlinear Absorbing Chromophores with Optical Limiting Properties at Telecommunication Wavelengths," Chemistry of Materials, 19(22), 5325-5335 (2007).

[13] P.-A. Bouit, K. Kamada, P. Feneyrou et al., "Two-Photon Absorption-Related Properties of Functionalized BODIPY Dyes in the Infrared Range up to Telecommunication Wavelengths," Advanced Materials, 21(10-11), 1151-1154 (2009).

[14] J. M. Hales, J. Matichak, S. Barlow et al., "Design of Polymethine Dyes with Large Third-Order Optical Nonlinearities and Loss Figures of Merit," Science, 327(5972), 1485-1488 (2010).

[15] R. L. Gieseking, S. Mukhopadhyay, C. Risko et al., "25th Anniversary Article: Design of Polymethine Dyes for All-Optical Switching Applications: Guidance from Theoretical and Computational Studies," Advanced Materials, 26(1), 68-84 (2014).

[16] S. Barlow, J.-L. Bredas, Y. A. Getmanenko et al., "Polymethine materials with solid-state third-order optical susceptibilities suitable for all-optical signal-processing applications," Materials Horizons, (2014).

[17] C. Cabanetos, W. Bentoumi, V. Silvestre et al., "New Cross-Linkable Polymers with Huisgen Reaction Incorporating High $\mu \beta$ Chromophores for Second-Order Nonlinear Optical Applications," Chemistry of Materials, 24(6), 1143-1157 (2012).

[18] W. Bentoumi, J.-C. Mulatier, P.-A. Bouit et al., "Concise Multigram-Scale Synthesis of Push-Pull Tricyanofuran-Based Hemicyanines with Giant Second-Order Nonlinearity: An Alternative for Electro-optic Materials," Chemistry - A European Journal, 20(29), 8909-8913 (2014).

[19] L. Wang, J. Jin, X. Chen et al., "A cyanine based fluorophore emitting both single photon near-infrared fluorescence and two-photon deep red fluorescence in aqueous solution," Organic \& Biomolecular Chemistry, 10(28), 5366-5370 (2012).

[20] A. Grichine, A. Haefele, S. Pascal et al., "Millisecond lifetime imaging with a europium complex using a commercial confocal microscope under one or two-photon excitation," Chemical Science, 5(9), 3475-3485 (2014).

[21] L. M. Tolbert, and X. Zhao, "Beyond the Cyanine Limit: Peierls Distortion and Symmetry Collapse in a Polymethine Dye," Journal of the American Chemical Society, 119(14), 3253-3258 (1997).

[22] X. Peng, F. Song, E. Lu et al., "Heptamethine Cyanine Dyes with a Large Stokes Shift and Strong Fluorescence: A Paradigm for Excited-State Intramolecular Charge Transfer," Journal of the American Chemical Society, 127(12), 4170-4171 (2005).

[23] J. M. Hales, S. Zheng, S. Barlow et al., "Bisdioxaborine Polymethines with Large Third-Order Nonlinearities for All-Optical Signal Processing," Journal of the American Chemical Society, 128(35), 11362-11363 (2006).

[24] P.-A. Bouit, C. Aronica, L. Toupet et al., "Continuous Symmetry Breaking Induced by Ion Pairing Effect in Heptamethine Cyanine Dyes: Beyond the Cyanine Limit,” Journal of the American Chemical Society, 132(12), 4328-4335 (2010).

[25] S. Dähne, "Color and Constitution: One Hundred Years of Research," Science, 199(4334), 1163-1167 (1978).

[26] S. R. Marder, C. B. Gorman, B. G. Tiemann et al., "Relation Between Bond-Length Alternation and Second Electronic Hyperpolarizability of Conjugated Organic Molecules," Science, 261(5118), 186-189 (1993).

[27] C. K. Chiang, C. R. Fincher, Y. W. Park et al., "Electrical Conductivity in Doped Polyacetylene," Physical Review Letters, 39(17), 1098-1101 (1977).

[28] F. Terenziani, A. Painelli, C. Katan et al., "Charge Instability in Quadrupolar Chromophores: Symmetry Breaking and Solvatochromism," Journal of the American Chemical Society, 128(49), 15742-15755 (2006). 
[29] F. Terenziani, O. V. Przhonska, S. Webster et al., "Essential-State Model for Polymethine Dyes: Symmetry Breaking and Optical Spectra," The Journal of Physical Chemistry Letters, 1(12), 1800-1804 (2010).

[30] J. D. Matichak, J. M. Hales, S. Barlow et al., "Dioxaborine- and Indole-Terminated Polymethines: Effects of Bridge Substitution on Absorption Spectra and Third-Order Polarizabilities," The Journal of Physical Chemistry A, 115(11), 2160-2168 (2011).

[31] L. G. S. Brooker, R. H. Sprague, C. P. Smyth et al., "Color and Constitution. I. Halochromism of Anhydronium Bases Related to the Cyanine Dyes1," Journal of the American Chemical Society, 62(5), 1116-1125 (1940).

[32] V. V. Egorov, "Nature of the optical transition in polymethine dyes and J-aggregates," The Journal of Chemical Physics, 116(7), 3090-3103 (2002).

[33] O. D. Kachkovsky, and D. M. Shut, "Electronic properties of polymethine systems. 12. Solitonic nature of charge distribution in the excited state," Dyes and Pigments, 71(1), 19-27 (2006).

[34] J. Fabian, "Symmetry-lowering distortion of near-infrared polymethine dyes—a study by first-principles methods," Journal of Molecular Structure: THEOCHEM, 766(1), 49-60 (2006).

[35] F. Würthner, R. Wortmann, R. Matschiner et al., "Merocyanine Dyes in the Cyanine Limit: A New Class of Chromophores for Photorefractive Materials," Angewandte Chemie International Edition in English, 36(24), 2765-2768 (1997).

[36] F. Würthner, G. Archetti, R. Schmidt et al., "Solvent Effect on Color, Band Shape, and Charge-Density Distribution for Merocyanine Dyes Close to the Cyanine Limit," Angewandte Chemie International Edition, 47(24), 4529-4532 (2008).

[37] U. Lawrentz, W. Grahn, K. Lukaszuk et al., "Donor-Acceptor Oligoenes with a Locked all-trans Conformation: Synthesis and Linear and Nonlinear Optical Properties," Chemistry - A European Journal, 8(7), 1573-1590 (2002).

[38] W. Leng, F. Würthner, and A. M. Kelley, "Solvent-Dependent Vibrational Frequencies and Reorganization Energies of Two Merocyanine Chromophores," The Journal of Physical Chemistry A, 109(8), 1570-1575 (2005).

[39] A. V. Kulinich, N. A. Derevyanko, and A. A. Ishchenko, "Electronic structure and solvatochromism of merocyanines based on N,N-diethylthiobarbituric acid," Journal of Photochemistry and Photobiology A: Chemistry, 188(2-3), 207-217 (2007).

[40] X.-H. Zhou, J. Luo, J. A. Davies et al., "Push-pull tetraene chromophores derived from dialkylaminophenyl, tetrahydroquinolinyl and julolidinyl moieties: optimization of second-order optical nonlinearity by fine-tuning the strength of electron-donating groups," Journal of Materials Chemistry, 22(32), 16390-16398 (2012).

[41] R. S. Lepkowicz, O. V. Przhonska, J. M. Hales et al., "Nature of the electronic transitions in thiacarbocyanines with a long polymethine chain," Chemical Physics, 305(1-3), 259-270 (2004).

[42] M. S. Briggs, D. D. Burns, M. E. Cooper et al., "A pH sensitive fluorescent cyanine dye for biological applications," Chemical Communications(23), 2323-2324 (2000).

[43] Y. Kim, S. A. Hilderbrand, R. Weissleder et al., "Sugar sensing based on induced pH changes," Chemical Communications(22), 2299-2301 (2007).

[44] S. A. Hilderbrand, and R. Weissleder, "Optimized pH-responsive cyanine fluorochromes for detection of acidic environments," Chemical Communications(26), 2747-2749 (2007).

[45] Y. Xu, Y. Liu, and X. Qian, "Novel cyanine dyes as fluorescent $\mathrm{pH}$ sensors: PET, ICT mechanism or resonance effect?," Journal of Photochemistry and Photobiology A: Chemistry, 190(1), 1-8 (2007).

[46] H. Lee, W. Akers, K. Bhushan et al., "Near-Infrared pH-Activatable Fluorescent Probes for Imaging Primary and Metastatic Breast Tumors," Bioconjugate Chemistry, 22(4), 777-784 (2011).

[47] S. Pascal, P.-A. Bouit, B. Le Guennic et al., "Symmetry loss of heptamethine cyanines: an example of dipole generation by ion-pairing effect," Proc. SPIE, 86220F-86220F (2013).

[48] K. Kiyose, H. Kojima, and T. Nagano, "Functional Near-Infrared Fluorescent Probes," Chemistry - An Asian Journal, 3(3), 506-515 (2008).

[49] M. Zhu, M. Yuan, X. Liu et al., "Visible Near-Infrared Chemosensor for Mercury Ion," Organic Letters, 10(7), 1481-1484 (2008).

[50] K. Kiyose, S. Aizawa, E. Sasaki et al., "Molecular Design Strategies for Near-Infrared Ratiometric Fluorescent Probes Based on the Unique Spectral Properties of Aminocyanines," Chemistry - A European Journal, 15(36), 9191-9200 (2009). 
[51] T. Myochin, K. Kiyose, K. Hanaoka et al., "Rational Design of Ratiometric Near-Infrared Fluorescent pH Probes with Various pKa Values, Based on Aminocyanine," Journal of the American Chemical Society, 133(10), 3401-3409 (2011).

[52] S. Pascal, A. Haefele, C. Monnereau et al., "Expanding the Polymethine Paradigm: Evidence for the Contribution of a Bis-Dipolar Electronic Structure," The Journal of Physical Chemistry A, 118(23), 4038-4047 (2014).

[53] L. Strekowski, M. Lipowska, and G. Patonay, "Substitution reactions of a nucleofugal group in heptamethine cyanine dyes. Synthesis of an isothiocyanato derivative for labeling of proteins with a near-infrared chromophore," The Journal of Organic Chemistry, 57(17), 4578-4580 (1992).

[54] L. Strekowski, J. C. Mason, M. Say et al., "Novel synthetic route to pH-sensitive 2,6-bis(substituted ethylidene)cyclohexanone/hydroxycyanine dyes that absorb in the visible/near-infrared regions," Heterocyclic Communications, 11(2), 129-134 (2005).

[55] J. H. Flanagan, S. H. Khan, S. Menchen et al., "Functionalized Tricarbocyanine Dyes as Near-Infrared Fluorescent Probes for Biomolecules," Bioconjugate Chemistry, 8(5), 751-756 (1997).

[56] F. Farooqui, M. A. Michael, and P. Reddy, [Efficient cyclic-bridged cyanine dyes] Patent, (2002). 